\title{
Zur letzten Eiszeit im alpinen und nordeuropäischen Raum
}

\begin{abstract}
On the last Glaciation of the Alps and Fennoscandia.

During the Pleistocene the Alps and the Fennoscandian Shield were covered several times with extensive ice caps. During the last Ice Age, the Würm or Weichsel Glaciation, the maximum extent of the glaciers occurred at the end of the Ice Age, as late as 20,000 years ago. The main retreat phases during deglaciation were marked as distinct ice marginal zones and dated between 20,000 and 9,000 BP.
\end{abstract}

\section{Key words:}

Würm/Weichsel glaciation, Late Würm, Late Glacial, interstadial, chronology, ice-marginal zone, moraine stratigraphy, retreat phases,

\section{Einleitung}

Während des Quartärs sind die Alpen und das skandinavische Gebirge mehrmals derart mit Eis bedeckt gewesen, daß sich das Eisregime bis ins Vorland ausgedehnt hat. Die alpine Gebirgsvergletscherung wird von zahlreichen ein Stück ins Vorland vorgedrungenen einzelnen Gletscherzungen charakterisiert, wogegen das skandinavische Inlandeis sich als ein umfangreicher Eiskuchen weit vom Vereisungszentrum im Gebirge ausgebreitet hat. Während der Eiszeiten herrschte zwischen dem alpinen und nordischen Eisschild in Mitteleuropa ein frostiges Periglazial-Klima.

Die Grundzüge der Eiszeitforschung, als Beispiel die alpine Vergletscherung, wurden von PENCK (1882) veröffentlicht. Die glazigenen Formen und Ablagerungen mit den vier Eiszeiten, Günz, Mindel, Riß und Würm, wurden von PENCK und BRÜCKNER (1901-1909) in ihrem umfangreichen dreibändigen Werk «Die Alpen im Eiszeitalter» ausführlich beschrieben. Wie J. GEIKIE (1895) auf den Britischen Inseln, so konnten PENCK (1879), WAHNSCHAFFE (1891) und KEILHACK (1896) auch am Südrande des skandinavischen Eisschildes mehrere Vorstöße unterscheiden, die KEILHACK den von ihm benannten Elster-, Saale- und Weichseleiszeit zuwies.

\section{Das Würmglazial im Alpenraum}

Das Würmglazial war die jüngste der vier großen, von PENCK erkannten, quartären Vereisungen. Die Gliederung der letzten Eiszeit erfolgte zunächst nach geomorphologischen Kriterien. Man unterschied die äußeren Endmoränen des Maximalstandes von den Endmoränen der Rückzugsphasen (PENCK 1882; PENCK \& BRÜCKNER 1901-1909; TROLL 1924). Heute geht man, wie schon die eben genannten Forscher, von einem einzigen kontinuierlichen Eisaufbau aus, der im Hochglazial vor etwa 20000 Jahren zur maximalen Ausführung führte. Während des Frühglazials, wo die Alpengletscher nur die zentralen Gebiete des Gebirges bedeckten und z. B. das Inntal bei Innsbruck vor 30000 bis 25000 Jahren eisfrei lag, kann man von keiner eigentlichen $\mathrm{Al}$ penvergletscherung sprechen.

Die Würmeiszeit wird im allgemeinen in das Früh-, Mittel- und Spätwürm eingeteilt. Aufgrund der Ausbreitung der Gletscher spricht man auch über das Früh-, Hoch- und Spätglazial. Stratigraphisch wurde das Würmglazial neulich auch in Unteres, Mittleres und Oberes Würm eingeteilt (CHALINE \& JERZ 1984). Die Gliederung des Frühwürms ist noch ungelöst. Als gesichert gilt ein relativ später Beginn der Hauptwürmzeit vor ca. 25000 Jahren und die nachfolgende Vorlandvereisung mit einem Maximum um 20000 bis 18000 Jahre v. h. Die Dauer der eigentlichen Vergletscherung betrug nur etwa 10000 Jahre.

Eine Gliederung der als Rückzugsstadien bezeichneten Wiedervorstöße wurde erstmals auch von PENCK \& BRÜCKNER (1909) nach den Typlokalitäten im Raume des eiszeitlichen Inngletschers erarbeitet. Ihre klassische und seither allgemein bekannte Dreigliederung «Bühl-Gschnitz-Daun» repräsentiert dabei wesentliche nochmalige Vorstoß-Phasen des spätglazialen Eisabbaues. Die Penck und Brücknersche Stadialabfolge bildet in den alpinen glazialgeologischen Arbeiten noch heute einen gül-

Gunnar Glückert, Prof., Institut für Quartärgeologie der Universität Turku, 20500 Turku, Finnland 


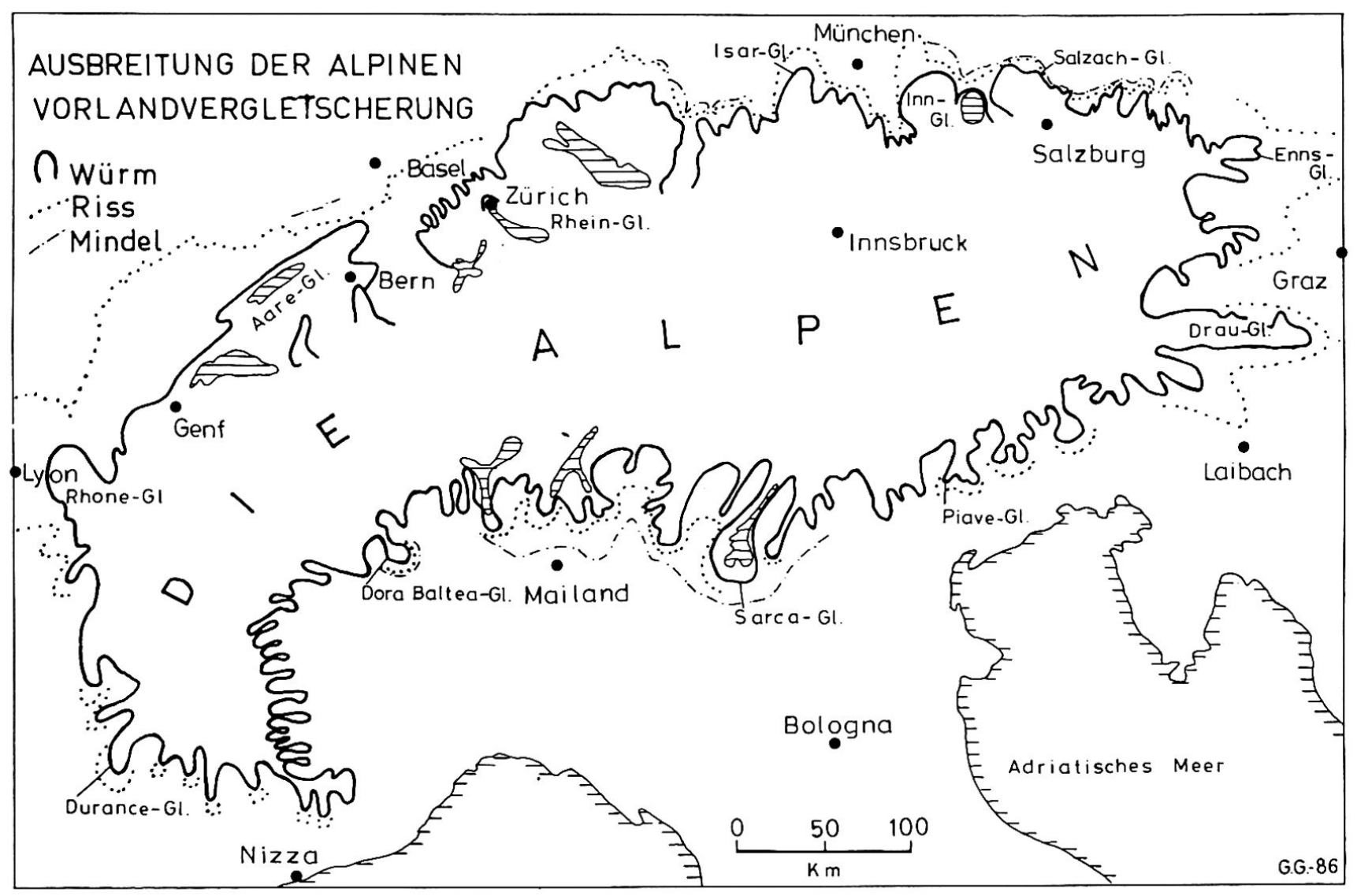

Abb. 1 Ausbreitung der alpinen Vorlandvergletscherungen während der Mindel-, Riß- und Würm-Eiszeit (nach versch. Quellen).

Fig. 1 The extent of the last Pleistocene glaciations of the Alps (from various sources).

tigen Ausgangspunkt für die Gliederung und Parallelisierung der Gletscherstände.

Die Gletscherstände im Hochglazial bilden bei den meisten Vorlandgletschern einen mehrfachen Endmoränenkranz, der die lokale maximale Ausdehnung der Würmvereisung vor etwa 20000 bis 18000 Jahren markiert. Die äußerste Würmeisrandlage des Inngletschers bildet z. B. drei markante Endmoränenbögen, die nach TROLL (1924) die Kirchseeoner, die Ebersberger und die Ölkofner Phase benannt sind.

Der bereits im ausgehenden Würm-Hochglazial einsetzende Eiszerfall nahm zu Beginn des Spätglazials zu. Im Alpenvorland rückten die Gletscherloben von ihren Zungenbecken umrahmenden äußersten Moränenbögen ab. Beim weiteren etappenweisen Rückschmelzen schalteten sich wiederholt Haltephasen mit kurzfristigen Vorstößen und entsprechenden Stadialmoränen ein. Die Bühl-Rückzugsphase und die Steinach-, Gschnitz-, Daun- und Egesen-Gletscherstände markieren selbständige lokale Gletschervorstöße, deren Eisrandlagen sowohl in Österreich und in der BRD als auch in der Schweiz deutlich zu sehen sind (z. B. KINZL 1932, HEUBER-
GER 1968, PATZELT 1972, V. HUSEN 1977, HANTKE 1978/ 80/83, PATZELT Und BORTENSCHLAGER 1978, WELTEN 1981 und MAISCH 1982; Tabelle 1).

Im Alpenraum gibt es auch Zeichen über die Ausbreitung der vorwürmeiszeitlichen Vergletscherungen. Nach den nur teilweise erhaltenen Gletscherablagerungen und Randlagen besa $\beta$ die Mindelvereisung die größte Ausbreitung während des Pleistozäns. Auch die äußersten Endmoränen der Rißvereisung liegen außerhalb des Maximalstandes der Würmgletscher (z. B. GLÜCKERT 1974; Abb. 1).

\section{Die Weichseleiszeit im skandinavischen Vereisungsgebiet}

Die jüngste Weichselvereisung Nordwesteuropas wird mit der Würmeiszeit der Alpen korreliert. Wie im Alpengebiet baute sich auch über Skandinavien ein bis über $2000 \mathrm{~m}$ Höhe angewachsener Eisschild auf. Dieser dehnte sich weit außerhalb des Gebirges, einerseits über die trockengefallene Nordsee bis zu den Vereisungszentren der Britischen Inseln, 


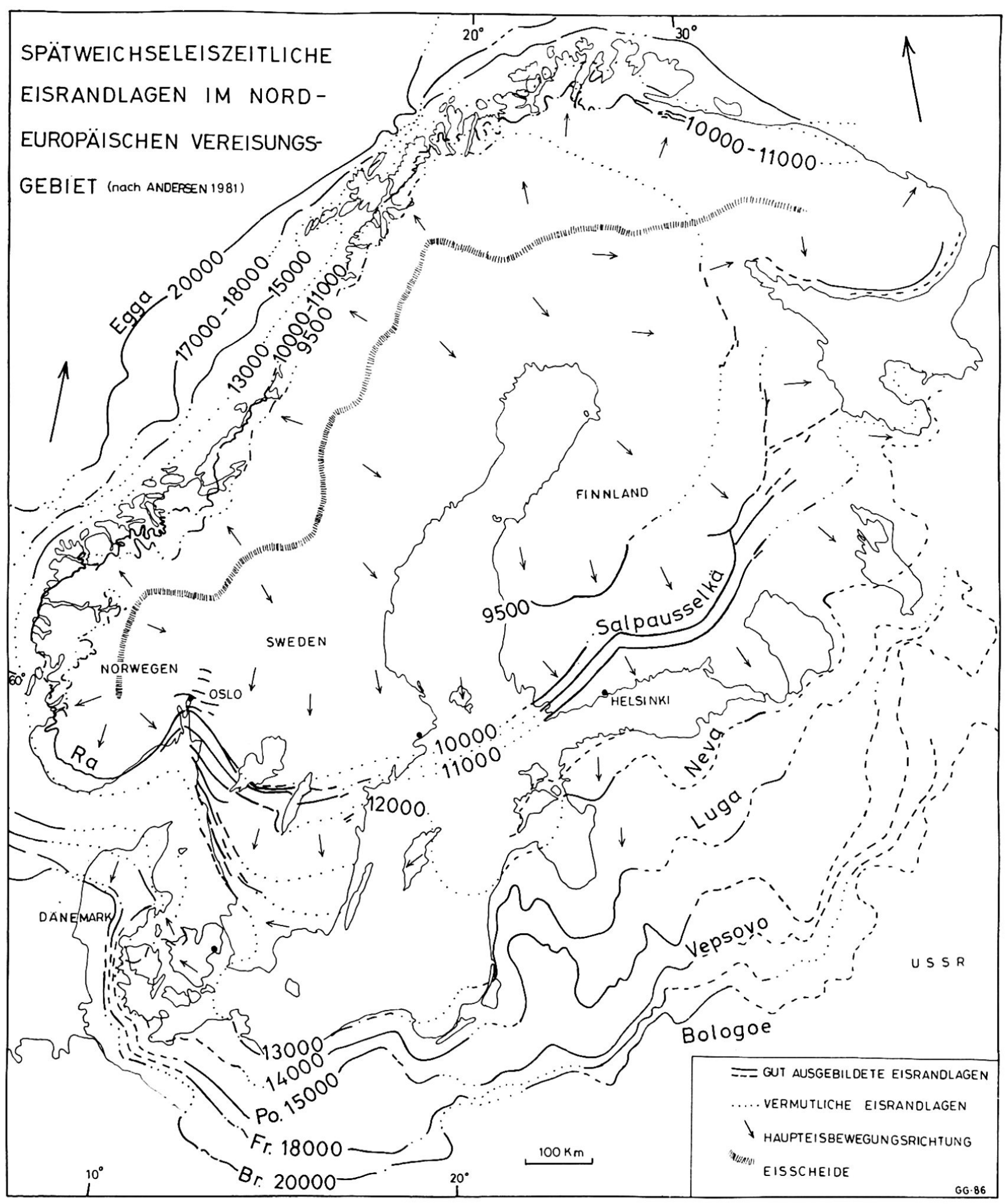

Abb. 2 Spätweichseleiszeitliche Eisrandlagen im nordeuropäischen Vereisungsgebiet (hauptsächlich nach Andersen 1981).

Fig. 2 Late Weichselian ice-marginal zones in northern Europe (mainly after Andersen 1981). 
andererseits über die Ostsee nach der Sowjetunion, Polen, Norddeutschland und Dänemark aus. Die maximale Ausdehnung des Eisschildes geschah erst am Ende der Eiszeit etwa vor 20000 Jahren (Abb. 2).

Die geologisch bedeutendsten hoch- und spätglazialen Eisrandlagen im skandinavischen Vereisungsgebiet können teilweise mit den entsprechenden Stadien im Alpenraum korreliert werden und sie sind in derTabelle 1 zusammengefaßt. Die Brandenburgerund die Frankfurter-Stadien sowie Pommern-, Rosenthal-, Rügen- und die Ra-Salpausselkä-Stadien sind zwischen 20000 und 10000 Jahren datiert worden. Sie sind auf der Karte in Abb. 2 dargestellt, die mit Hilfe der Zusammenfassung von Andersen (1981) über den Eisrückzug in verschiedenen Teilen des Vereisungsgebiets zusammengestellt ist .

Die ausgeprägteste Eisrandlage in Skandinavien ist ohne weiteres die zwei- bis dreifache Ra-Salpausselkär-Eisrandzone aus der Jüngeren Dryaszeit, die Fennoskandien umrahmt. Die Lage dieser Eisrandzone scheint glaziklimatisch bedingt zu sein. Ihre großen Bögen markieren die Grenzen von aktiven Loben des Inlandeises, das teilweise in die ehemalige Ostsee endete (z. B. LEIVISKÄ 1920, SAURAMO 1929, 1958, VIRKKALA 1963, NIEMELÄ 1971 und GLÜKKERT 1986).

Diese morphologisch gewaltige dryaszeitliche Eisrandlage besteht aus breiten glazifluviatilen marginalen Deltas und aus kleineren Rücken (Endmoränen). Spuren von der Glazialtektonik gibt es wenig. Im allgemeinen betrachtet man die Eisrandlage als Satzendmoränen, im Gegensatz zu den markanten Stauchendmoränen Norddeutschlands (z. B. LEIVISKÄ 1920, GRIPP 1964, GLÜCKERT 1973, 1977. 1986).

Wie im Alpenraum hat man auch in Skandinavien wenig Kenntnisse über das glazigene Geschehen während der Frühweichseleiszeit, z. B. die exakte Gliederung der Eiszeit ist noch offen. Die Moränenstratigraphie in Nordfinnland läßt etwas über die Ausbreitung der Gletscher während des Spätquartärs im nördlichen Teil Fennoskandiens vermuten. Bei den Moränenuntersuchungen wurden auch eine Anzahl submoränaler Sand- und Siltschichten sowie organogene Ablagerungen entdeckt, von denen jedoch ein Teil umgelagert ist (z. B. HIRVAS \& NENONEN 1987; Abb. 3).

Die organisches Material enthaltenden Funde sind durch Pollenanalyse und mit der ${ }^{\mathrm{H}} \mathrm{C}$-Methode datiert worden. Die Altersbestimmungen liegen meist über 50000 Jahren oder zwischen 40000 und 50000 Jahren v. h. (Weichsel-Interstadial) (z. B. KORPELA 1969, DONNER 1983).

Sowohl in Nordfinnland als auch in Nordschweden hat man auf den liegenden Eem-Interglazialablagerungen zwei separate Grundmoränenschichten der letzten Eiszeit entdeckt, die oft durch ein Zwischenlager von minerogenen oder organischen Sedimen- ten eines Interstadiales getrennt sind. Weiter im Süden, in Mittelfinnland, fehlt die untere Moränenschicht, und die Weichselablagerungen bestehen nur aus einem Moränenlager. Das Eis hat während der Frühweichseleiszeit nur Nordfinnland und das

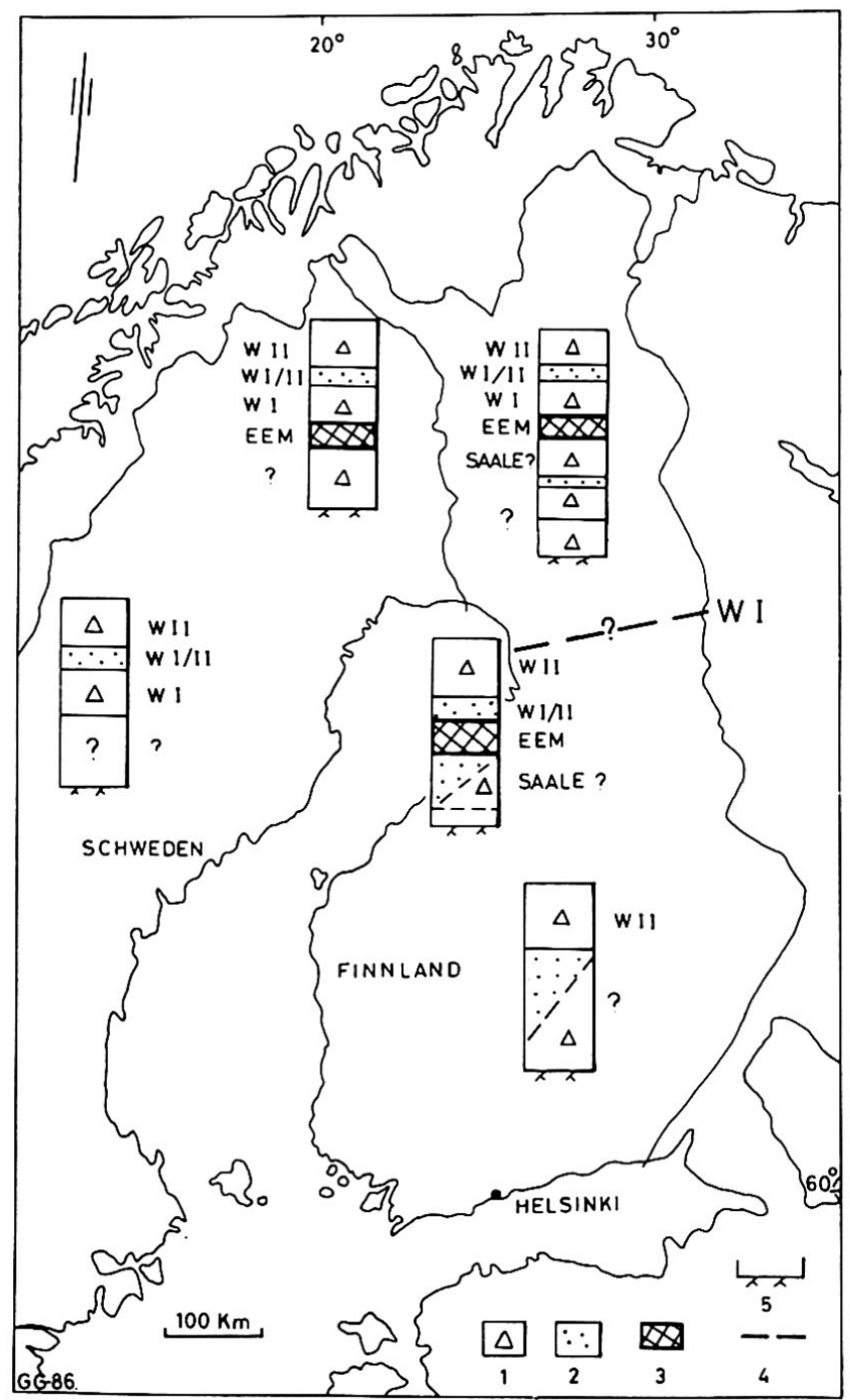

Abb. 3 Moränenstratigraphie im nördlichen Fennoskandien und die vermutliche Verbreitung der Frühweichselvereisung (WI). 1 = Grundmoräne, 2 = Submoränale minerogene Sedimente, 3 = Organische Sedimente, 4 = Vermutl. Ausbreitung der Frühweichselvereisung (W I), $5=$ Felsen (Grundgebirge).

Fig. 3 Till stratigraphy in northern Fennoscandia and the extent (?) of the Early Weichselian ice. 1 = basal till, 2 = submorainic minerogenic sediments, $3=$ organic sediments, $4=$ the probable extent of the Early Weichselian ice (W I), 5 = bedrock. 
Tabelle 1 Chronologisch korrelierbare Eisrandzonen im Bereich des alpinen und nordeuropäischen Vereisungsgebiets während der Spätweichsel-Mürm-Eiszeit (nach versch. Autoren).

Table 1 Chronologically correlated Late Weichselian ice-marginal zones in the areas of the Alpine and Scandinavian Ice Sheets (several authors).

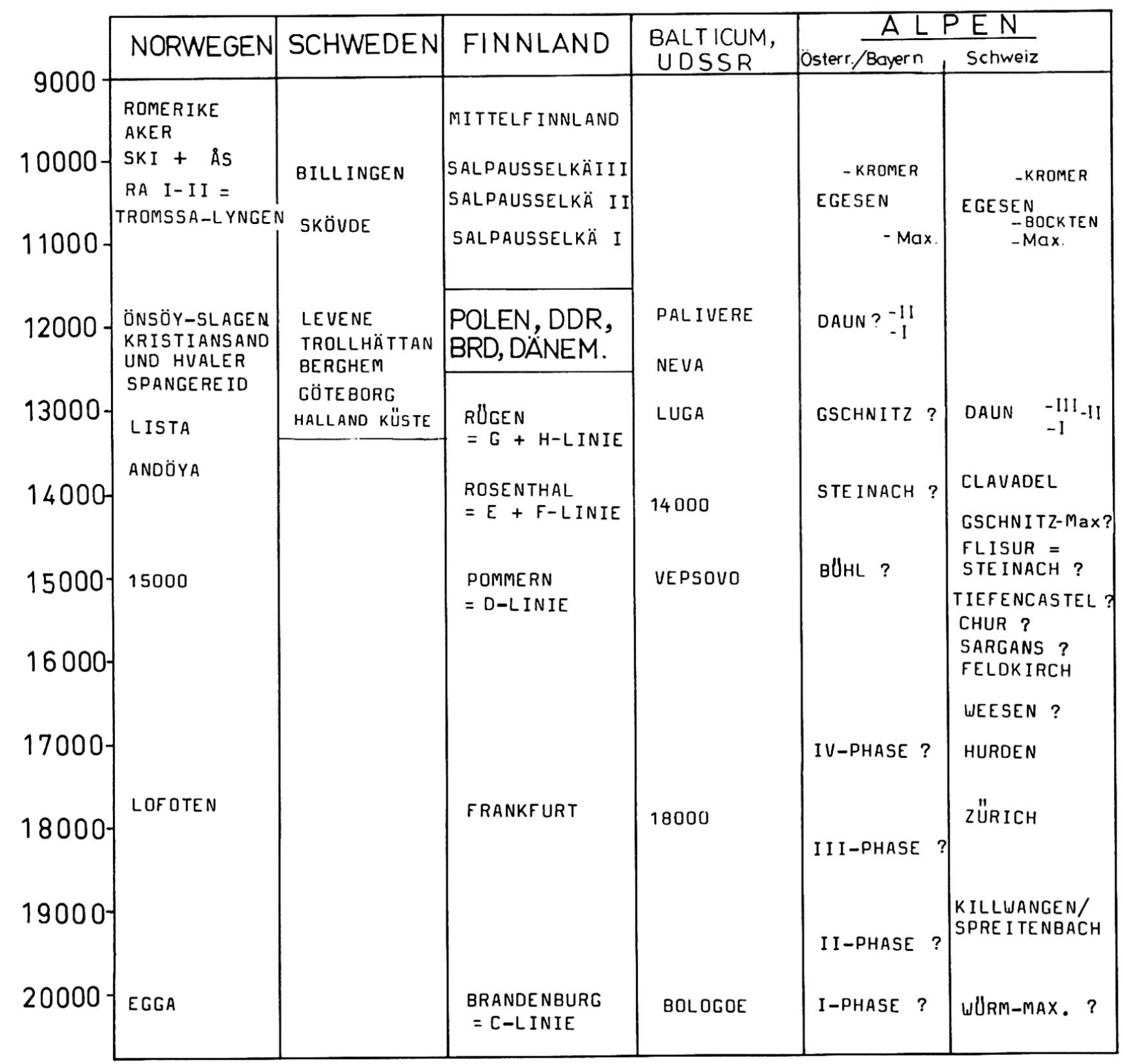

skandinavische Gebirge bedeckt (z. B. LUNDQVIST 1967, KORPELA 1969, DONNER 1983, HIRVAS \& NENO. NEN 1987; Abb. 3).

Das glazigene Material und die Formen in Fennoskandien unterscheiden sich erheblich von den entsprechenden Funden sowohl am Südrand der skandinavischen Vereisung als auch im Alpenraum. Umfangreiche moorreiche Moränengebiete, zahlreiche große Drumlinfelder, Endmoränenscharen und lange, hohe Oszüge charakterisieren die zentralen Teile des fennoskandischen Vereisungsgebiets. Das abgerundete Grundgebirge mit unzähligen eisgeschliffenen Rundhöckern, besonders in den felsenreichen Küsten- und Seengebieten in Schweden und Finnland, zeugen von einer intensiven Glazialerosion im Laufe des Pleistozäns. 


\section{Literatur:}

ANDERSEN, B. G. (1981): Late Weichselian Ice Sheets in Eurasia and Greenland. In: Denton \& Hughes: The Last Great Ice Sheets, 1-65.

CHALINE, J. \& JERZ, H. (1984): Arbeitsergebnisse der Subkommission für Europäische Quartärstratigraphie. Stratotypen des Würm-Glazials. Eiszeitalter u. Gegenwart 34, 185-206, Hannover.

DONNER, J. J. (1983): The identification of Eemian interglacial and Weichselian interstadial deposits in Finland. Ann Acad. Sci. Fennical, A III. 136, 38 S.

GEIKIE, J. (1895): Classification of European Glacial Deposits. Journal of Geology, 3/3, Chicago.

GLÜCKERT, G. (1973): Glazialmorphogenese der weichseleiszeitlichen Moränen des Eckernförder Zungenbeckens (Schleswig-Holstein). In: Meyniana, 23, 19-48, Kiel.

GLÜCKERT, G. (1974): On Pleistocene glaciations in the German alpine foreland. In: Bull. Geol. Soc. Finland, 46, 117-131.

GLÜCKERT, G. (1977): On the Salpausselkä ice-marginal formations in southern Finland. In: Zeitschr. f. Geomorph. N. F. Suppl.-Bd. 78-88, Berlin-Stuttgart.

GLÜCKERT, G. (1986): The First Salpausselkä at Lohja, southern Finland. In: Bull. Geol. Soc. Finland, 58, 1, 45-55.

GRIPP, K. (1964): Erdgeschichte von Schleswig-Holstein, 411 S., Neumünster.

HANTKE, R. (1978/80/83): Eiszeitalter. 3 Bände, 1931 S., Thun.

HEUBERGER, H. (1968): Die Alpengletscher im Spät- und Postglazial. Eiszeitalter und Gegenwart 19, 270-275, Öhringen.

HIRVAS, H. \& NENONEN, K. (1987): The till stratigraphy of Finland, Geol. Surv. Finland., Spec. Paper 3 (im Druck)

HUSEN, D. van (1977): Zur Fazies und Stratigraphie der jungpleistozänen Ablagerungen im Trauntal. In: Jahresh. Geol. B.-Anst. 120, 1, 130 S., Wien.

KEILHACK, K. (1896): Die Geikische Gliederung der nordeuropäischen Glacialablagerungen. Jahrb. preuss. geol. Landesanstalt, 16, Berlin.

KINZL, H. (1932): Die größten nacheiszeitlichen Gletschervorstöße in den Schweizer Alpen und in der Mont-BlancGruppe. In: Zeitschr. f. Gletscherk., Bd. XIX, 4-5, 269-397.
KORPELA, K. (1969): Die Weichsel-Eiszeit und inr Interstadial in Peräpohjola (nördliches Nordfinnland) im Lichte von submoränen Sedimenten. In: Ann. Acad. Sci. Fenn., A, III, 99, $108 \mathrm{~S}$

LEIVISKÄ, I. (1920): Der Salpausselkä. Fennia 41, 3, 389 S.

LUNDQVIST, J. (1967): Submoräna sediment i Jämtlands län. In: Sveriges Geol. Unders., Ser. C, 618, 267 S.

MAISCH, M. (1982): Zur Gletscher- und Klimageschichte des alpinen Spätglazials. In: Geographica Helvetica 37, 2 93-104.

NIEMELÄ, J. (1977): Die quartäre Stratigraphie von Tonablagerungen und der Rückzug des Inlandeises zwischen Helsinki und Hämeenlinna in Südfinnland. Bull. Comm. géol. Finlande 253, $79 \mathrm{~S}$

PATZELT, G. (1972): Die spätglazialen Stadien und postglazialen Schwankungen von Ostalpengletschern. In: Ber. der. dt. Botan. Ges., Bd. 85, 1-4.

PATZELT, G. und BORTENSCHLAGER, S. (1978): Spät- und nacheiszeitliche Gletscher- und Vegetationsentwicklung im inneren Ötztal. In: Innsbrucker Raum und Ötztal: Führer zur Tirol-Exkursion anl. der DEUQUA, 13-25.

PENCK, A. (1879): Die Geschiebeformation Norddeutschlands. Zeitschr. d. deutsch. Geol. Gesellschaft, 31.

PENCK, A. (1882): Die Vergletscherung der Deutschen Alpen. 483 S., Leipzig.

PENCK, A. und BRÜCKNER, E. (1909): Die Alpen im Eiszeitalter. 3. Bände, 1199 S., Leipzig.

SAURAMO, M. (1929): The Quaternary Geology of Finland. Bull. Comm. géol. Finlande, 86, $110 \mathrm{~S}$.

SAURAMO, M. (1958): Die Geschichte der Ostsee. In: Ann. Acad. Sci. Fenn., A, III, 51, 522 S.

TROLL, K. (1924): Der diluviale Inn-Chiemsee-Gletscher Forsch. d. Deutsch. Landes- und Volkskunde, 23/1, 121 S., Stuttgart.

VIRKKALA, K. (1963): On the ice-marginal formations in southwestern Finland. Bull. Comm. géol. Finlande, 210, 76 S.

WAHNSCHAFFE, F. (1891): Die Ursachen der Oberflächengestaltung des norddeutschen Flachlandes, Stuttgart.

WELTEN, M. (1981): Gletscher und Vegetation im Lauf der letzten hunderttausend Jahre. Vorläufige Mitteilung. In: Gletscher und Klima. Jahrb. d. Schweiz. Nat.-Gesellschaft, wissenschaftl. Teil: 5-18, 1978. 